\title{
Towards Optimized Efficiency of Ablation in Copper using a 515nm Picosecond Laser Process
}

\author{
Dongsig Shin ${ }^{* 1}$, Yongkwon Cho ${ }^{* 1}$ and Hyonkee Sohn ${ }^{* 1}$ \\ ${ }^{* 1}$ Korea Institute of Machinery \& Materials, 156 Gajeongbuk-Ro, Yuseong-gu, \\ DaeJeon, 305-343, South Korea \\ E-mail:dsshin@kimm.re.kr
}

\begin{abstract}
A shorter laser pulse provides smaller heat affected zones and higher ablation accuracy, which are demanded for metal micro-fabrication processes. In particular, picosecond laser systems, which are suitable for operation in industrial environments, are of great interest for many practical applications. However, low productivity has been a limitation to broadening their industrial application. It was recently reported that when micromachining copper with a $1064 \mathrm{~nm}$ picosecond laser, a desirable pulse energy and repetition rate exist for achieving the maximum volume ablation rate. In this paper, we used a $515 \mathrm{~nm}$ picosecond laser, which is more efficient for micromachining copper in terms of laser energy absorption, and determined its optimum pulse energy and repetition rate. A theoretical analysis based on the experimental data on copper ablation showed that using a $515 \mathrm{~nm}$ picosecond laser instead of a $1064 \mathrm{~nm}$ picosecond laser is favorable in that the calculated threshold fluence is $75 \%$ lower and the optical penetration depth is $50 \%$ greater.
\end{abstract}

DOI: $10.2961 /$ jlmn.2015.03.0001

Keywords: picosecond laser, volume ablation rate, copper, threshold fluence, penetration depth

\section{Introduction}

Recent advances in the development of ultra-short pulse lasers are making such devices ever more accessible to industries. In particular, ultra-short pulse high-power lasers can now implement non-thermal processing and precision machining at the same time. On this basis, this process has begun to be effectively and broadly applied industrially $[1,2]$. At this point, many studies still use an infrared-based ultra-short pulsed laser for patterning [3-6] although a shorter wavelength laser beam can produce much higher average power than before.

In this paper, the $515 \mathrm{~nm}$ wavelength of an ultra-short pulse laser was employed to verify its ablation efficiency for patterning copper based on an infrared based theoretical study $[3-5,7]$. The purpose of this study is to determine the optimized process parameters, such as fluence and repetition rate, for achieving optimized process efficiency for the ablation of copper using this $515 \mathrm{~nm}$ wavelength ultra-short pulse laser beam process.

The results of this study suggest an optimum solution for the micro-machining processes of various metals including copper using a $515 \mathrm{~nm}$ wavelength ultra-short pulsed laser.

\section{Experimental Setup}

Copper is the main material used for printing rolls, and previous studies have shown that the reflectance of copper is reduced by more than half when it is irradiated in the green wavelength $(515 \mathrm{~nm})$ as compared with the nearinfrared wavelength $[3-5,7,8]$. This means that when a laser beam in the green wavelength is used, the absorption rate is increased by two-fold or more, which leads to further improvement of the ablation efficiency.

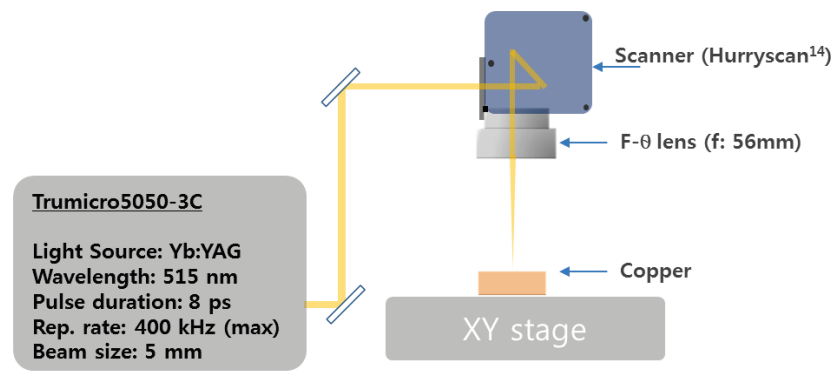

Fig. 1 Schematic illustration of experimental setup for laser ablation process.

In this paper, in order to find the optimum pulse energy for processing copper using an ultra-short laser (Trumpf, Trumicro5050-3C, Germany) as described above, parallel experimental and theoretical approaches were integrated. In order to obtain the correlation between the volume ablation rate $(\mathrm{dV} / \mathrm{dt})$ and the fluence $(\mathrm{F})$ of the laser beam, the diameter and ablation depth of the crater after processing as well as the focal spot size of the laser beam $\left(r_{d}\right)$ are required. A non-contact three-dimensional fine shape measuring device (Nanosystem, NV-E1000, Korea) was used to measure the diameter and ablation depth of the produced crater, for determining the correlation between the optical focal size and the measured diameter and depth of the crater, the induced penetration depth, and the threshold fluence. Finally, experiments have been conducted on copper surface patterning.

\section{Theoretical background}

The volume ablation rate $(\mathrm{dV} / \mathrm{dt})$ was determined as delineated in Eq. (1) [9, 10], which is composed of the average power $\left(\mathrm{P}_{\mathrm{av}}\right)$, pulse repetition rate (Rep.), threshold flu- 
ence $\left(\mathrm{F}_{\mathrm{th}}\right)$ and penetration depth $(\delta)$. Additionally, the threshold fluence $\left(\mathrm{F}_{\text {th }}\right)$ and the absorption coefficient $(1 / \alpha)$ were determined by Eq. (2) and Eq. (3) [5, 11-13]; these equations can be applied according to sequence as shown in Fig. 2.

$$
\begin{aligned}
& \frac{d V}{d t}=\frac{1}{4} \cdot \pi \cdot w_{0}^{2} \cdot \delta \cdot \operatorname{Rep} \cdot \ln ^{2}\left(\frac{2 \cdot P_{a v}}{\operatorname{Rep} \cdot \pi \cdot w_{0}^{2} \cdot F_{t h}}\right) \\
& F_{t h}=F \exp \left(-r_{d}^{2} / 2 w_{0}^{2}\right) \\
& L=\frac{1}{\alpha} \ln \left(\frac{F}{F_{t h}}\right)=\delta \ln \left(\frac{F}{F_{t h}}\right)
\end{aligned}
$$

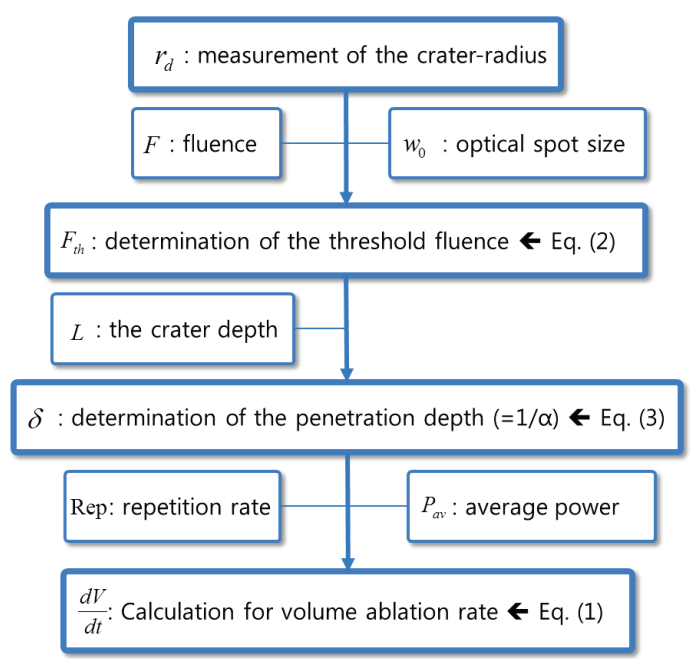

Fig. 2 Experimental design based on the equation for calculated volume ablation rate.

According to Eq. (2), to find the threshold fluence $\left(\mathrm{F}_{\mathrm{th}}\right)$, the radius of the optical focal point $\left(\mathrm{w}_{0}\right)$ and the radius of the crater $\left(r_{d}\right)$ are required during processing. The applied optical spot size on the focal point was $14.56 \mu \mathrm{m}$. The diameter of the crater was measured using a non-contact three-dimensional fine shape measuring device and an optical microscope.

\section{Results and Discussion}

\subsection{Dependency of the crater diameter and the thresh- old fluence}

Figure 3 shows the copper surface after the laser beam irradiation, which was performed under a fluence of 0.28 $\mathrm{J} / \mathrm{cm}^{2}$ and 200 pulses. Figure 4 shows the change in the central diameters of the craters produced in copper based on the increase of the total input energy, which is defined by multiplying the fluence and the number of pulses. Depending on the total input energy, the diameter of the crater is shown to finally converge after increasing in the initial stage. The value of each converging diameter increases depending on the fluence of the laser beam. This reveals that the crater size on the material varies according to the pulse energy of the laser beam under the same optical spot size. Equation (2) shows the relationship between the di- ameter of the crater and the optical focal spot size, which is used to derive the threshold fluence.

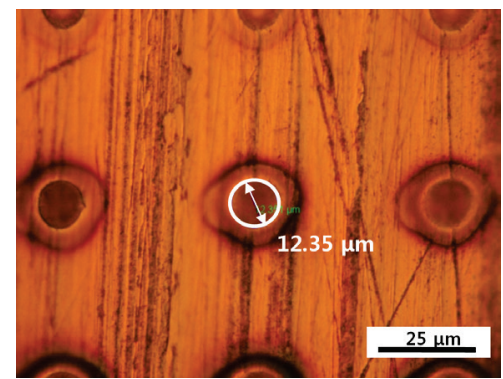

Fig. 3 Optical microscope image of the copper surface patterned under the following conditions: $\mathrm{F}=$ $0.28 \mathrm{~J} / \mathrm{cm} 2, \mathrm{~N}=200$ pulses

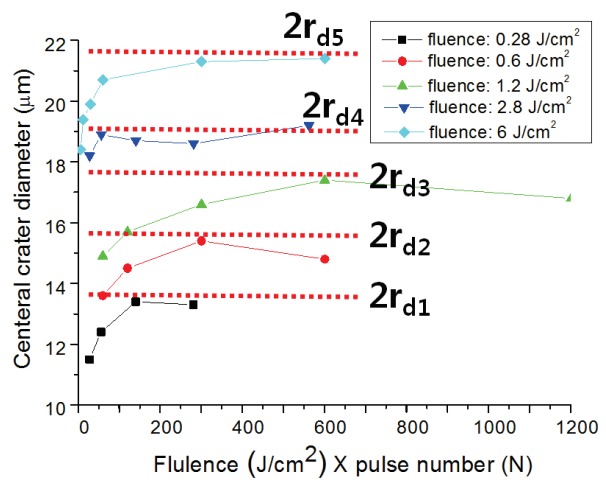

Fig. 4 Change in the central diameters of craters produced in copper according to the total input energy (i.e., fluence $\times$ number of pulses), $\mathrm{N}=200$ pulses

In the previous section, we introduced the radius of the crater $\left(r_{d}\right)$, which is a component of Eq. (2) and is used to determine the threshold fluence $\left(\mathrm{F}_{\text {th }}\right)$. Figure 5 presents a comparison of the converged crater diameters in copper obtained from experiments and simulations with an increase of the incident laser fluence $(\mathrm{F})$ and the threshold fluence $\left(\mathrm{F}_{\mathrm{th}}\right)$. The experimental results agree well with the simulations results at a threshold fluence of $0.075 \mathrm{~J} / \mathrm{cm}^{2}$ as expressed by the solid line. This shows that the threshold fluence $\left(\mathrm{F}_{\text {th }}\right)$ at the wavelength of $515 \mathrm{~nm}$ was smaller than a quarter of that at a wavelength of $1064 \mathrm{~nm}$ [4].

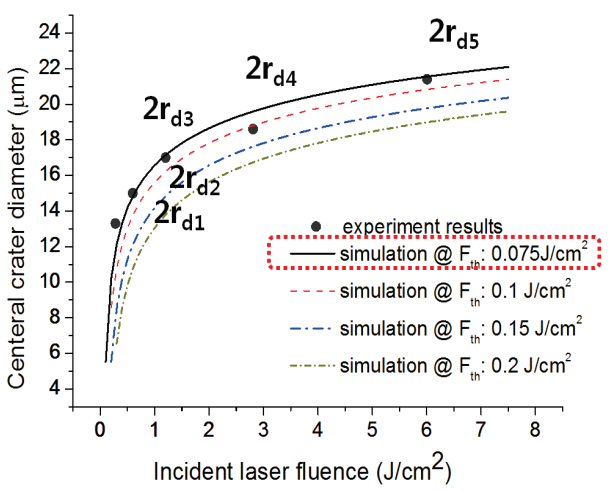

Fig. 5 Comparison of the converged central crater diameters of copper obtained from experiments and simulations. 


\subsection{Penetration depth}

The volume ablation rate $(\mathrm{dV} / \mathrm{dt})$ represented in Eq. (1) has a functional relation with the driven threshold fluence $\left(\mathrm{F}_{\text {th }}\right)$ and the penetration depth $(\delta=1 / \alpha)$, as shown in Eq. (3). That is, it is possible to determine the penetration depth by substituting the function of the fluence and the ablation depth per pulse.

Figure 6 shows a cross-sectional profile of a crater that was processed under the conditions of 200 pulses and a fluence $(F)$ of $0.28 \mathrm{~J} / \mathrm{cm}^{2}$. The ablation depth was $12.2 \mu \mathrm{m}$ and the processing depth per pulse (L) was $61 \mathrm{~nm}$. Here, the derived penetration depth $(\delta)$ result is $46 \mathrm{~nm}$ after using the fluence $(\mathrm{F})$, the processing depth per pulse $(\mathrm{L})$ and the threshold fluence $\left(\mathrm{F}_{\text {th }}\right)$ derived in the previous section by substituting Eq. (3). This indicates there is an increase in depth of $50 \%$ or more compared to the penetration depth of $30 \mathrm{~nm}$, which was determined at a wavelength of 1064 $\mathrm{nm}$ [4]. The deeper penetration depth in the case of using the green wavelength $(515 \mathrm{~nm})$ can be ascribed to its higher absorption rate as compared with the near-infrared wavelength $(1064 \mathrm{~nm})$. This is intimately linked with that the threshold flunce $\left(\mathrm{F}_{\mathrm{th}}\right)$ at the wavelength of $515 \mathrm{~nm}$ was smaller than a quarter of that at a wavelength of $1064 \mathrm{~nm}$.

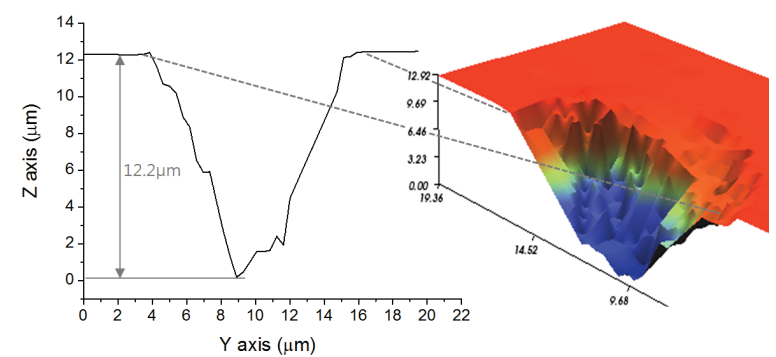

Fig. 6 Cross-sectional profile of a drilled crater oncopper surface after laser beam radiation under the following condition: $\mathrm{F}=0.28 \mathrm{~J} / \mathrm{cm}^{2}, \mathrm{~N}=200$ pulses.

\subsection{Dependency of volume ablation rate}

In our previous work, Equation (1) was defined in order to maximize the volume ablation rate by deriving the penetration depth $(\delta)$ and the threshold fluence $\left(\mathrm{F}_{\text {th }}\right)$ by experimental verification. Consequently, we could predict the volume ablation rate with an increase of repetition rate.

Figure 7 shows a relative comparison between the simulated ablation rate and arbitrary ablation depth as determined by the experiment with an increase of the repetition rate and the average power.

Figure 8 shows the three-dimensional profile of a surface that has been ablated under conditions referred to as (a) and (b) in Fig.7. Here, the relative comparison for ablation depth shows the ratio of the ablation depth when the maximum depth is 1 under conditions of an average power of $3.2 \mathrm{~W}$ and a repetition rate of $400 \mathrm{kHz}$. When the ablation depth of $150.5 \mu \mathrm{m}$ for the conditions marked (a) is 1 , the ablation depth of $109.2 \mu \mathrm{m}$ becomes 0.72 for the conditions marked (b), as shown in Fig. 8. At this point, the experimental results show that the parameters used in Fig .7 (b) are more efficient than those of Fig. 7 (a). Additionally, the ablation rate rises and falls at some point with an increase of the repetition rate under each average power.

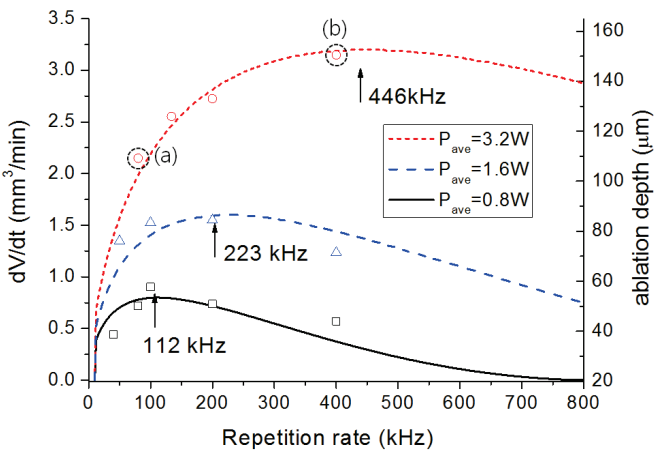

Fig. 7 Variation in the volume ablation rate and arbitrary ablation depth of copper as a function of the repetition rate under the conditions of wavelength: 515 $\mathrm{nm}$, pulse duration: $8 \mathrm{ps}$, spot size: $57.4 \mu \mathrm{m}$ and scan speed: $1 \mathrm{~m} / \mathrm{s}$ (dotted-dashed line: calculated volume ablation rate, symbol: arbitrary ablation depth).
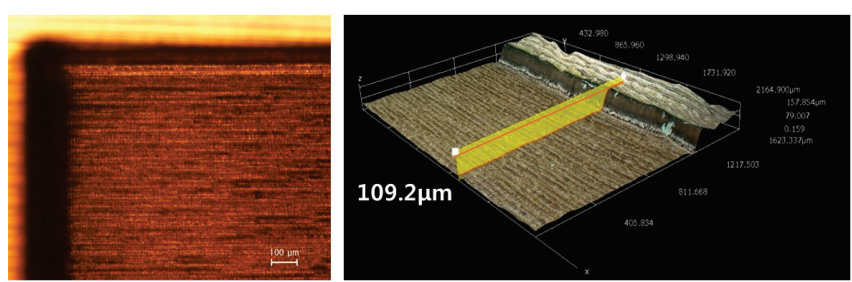

(a)
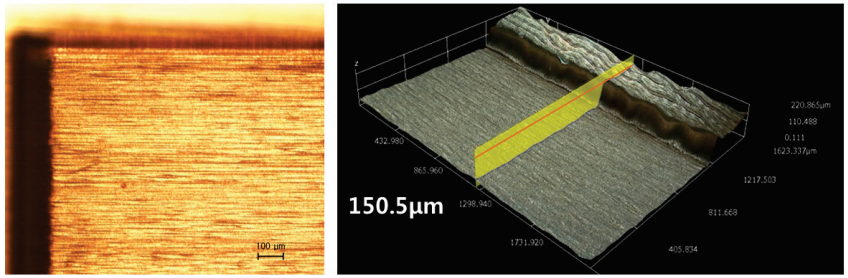

(b)

Fig. 8 Pictures and 3D profiles of ablated layer of copper under the conditions marked as (a) and (b) in Fig. 7.

Here, the highest ablation rate can be obtained in the region where the efficiency of the processing is maximized. Moreover, each average power has a corresponding point for the highest ablation rate with an increasing repetition rate. That is, average power of $3.2 \mathrm{~W}$ has a maximum ablation rate at a $446 \mathrm{kHz}$ repetition rate, while with $1.6 \mathrm{~W}$ and $0.8 \mathrm{~W}$ average power, the respective highest points are reached under repetition rates of $223 \mathrm{kHz}$ and $112 \mathrm{kHz}$.

This shows that the repetition rate is accompanied by a proportional increase of the average power. That is, the pulse energy, which is determined by dividing the average power by the repetition rate, remains constant. In consequence, the pulse energy is $7.17 \mu \mathrm{J}$. The fluence, which is defined by the pulse energy per unit area, is $0.28 \mathrm{~J} / \mathrm{cm}^{2}$. This is also smaller than a quarter of that at a wavelength of $1064 \mathrm{~nm}$ as shown in a prior study [4].

\subsection{Patterning process using an optimized fluence}

Figure 9 shows the embossed pattern on a copper block produced using a laser beam with a fluence of $0.28 \mathrm{~J} / \mathrm{cm}^{2}$, which is the optimum condition in terms of efficiency obtained from the above experiment. The experimental result 
verified that this cold ablation process could be achieved because the fluence for the maximum volume ablation rate is only four times the threshold fluence. However, there is a limitation in terms of productivity, because the average power was only $0.75 \mathrm{~W}$ for inducing a fluence of 0.28 $\mathrm{J} / \mathrm{cm}^{2}$ under the limited repetition rate of $100 \mathrm{kHz}$. In order to increase productivity, it is necessary to increase the average power and repetition rate proportionally. For example, the repetition rate is required to be $1 \mathrm{MHz}$ in the case of an average power of $7.5 \mathrm{~W}$ under the same fluence and pulse energy.

At this point, when using extremely high repetition, heat accumulation becomes a concern. It is thus necessary to use faster scanning techniques to minimize the thermal effects under a higher repetition rate. This topic will be introduced in a future study.

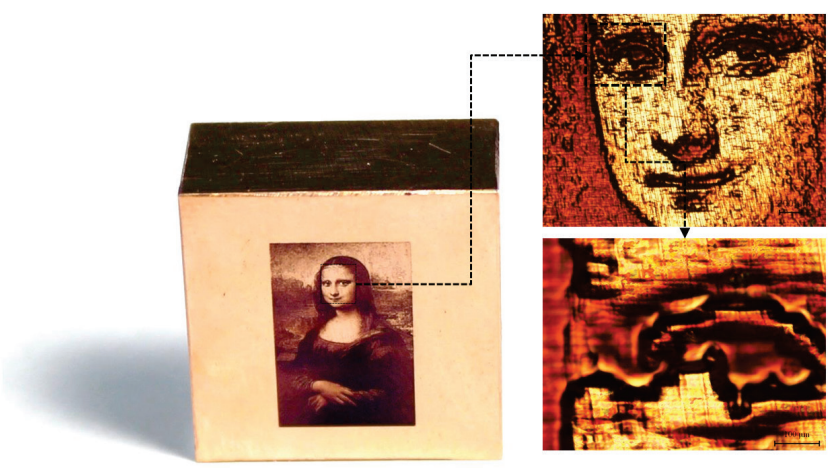

Fig. 9 Pictures of the embossed pattern of the Mona Lisa on a copper block under the following conditions; wavelength: $515 \mathrm{~nm}$, pulse duration: $8 \mathrm{ps}$, reptition rate: $100 \mathrm{kHz}$, spot diameter: $57.4 \mu \mathrm{m}$ and fluence: $0.28 \mathrm{~J} / \mathrm{cm}^{2}$.

\section{Conclusions}

This research attempted to verify the ablation efficiency of an ablation process on copper using a $515 \mathrm{~nm}$ wavelength ultra-short pulse laser. A theoretical analysis based on the obtained copper ablation experimental data showed that using a $515 \mathrm{~nm}$ picosecond laser instead of a $1064 \mathrm{~nm}$ picosecond laser is favorable. Specifically, the calculated threshold fluence is $75 \%$ lower and the optical penetration depth is $50 \%$ greater with the $515 \mathrm{~nm}$ picosecond laser. Finally, we were able to obtain a completely neat and clean micro-patterned surface based on optimized parameters determined for the $515 \mathrm{~nm}$ picosecond laser. However, in order to increase productivity, it will be necessary to increase the average power and the repetition rate proportionally and use faster scanning techniques to minimize the thermal effects under the higher repetition rate. This topic will be introduced in a future study.

\section{ACKNOWLEDGEMENT}

This work was supported by the Korea Research Council for Industrial Science \& Technology as part of the project "Mass-Production Technology Support Center for Green Energy Devices" and "Printing Roll Micro-pattering based on the Scanner and Multi-beams using DOE".

\section{References}

[1] Hennig, G., Selbmann K.H., and Brüning, S.: Laser Technik Journal, Vol. 5, (2008) 52.

[2] Bruening, S., Hennig, G, Eifel, S and Gillner, A.: Physics Procedia, Vol. 12, (2011) 105.

[3] Neuenschwander, B., Guido, F., Nussbaum, C. and Joss, B.: Proc. SPIE. Vol. 7584, (2010) 75840R.

[4] Schmid, M., Neuenschwander, B., Romano, V.: Proc. SPIE. Vol. 7920, (2011) 792009.

[5] Jandeleit, J., Urbasch, G., Hoffmann, H.D., Treusch, H. and G., Kreutz, E.W.: Applied Physics A, Vol. 63, (1996) 117.

[6] Cheng, J., Perrie, W., Edwardson, S.P., Fearon, E., Dearden, G. and Watkins, K.G.: Applied Surface Science, Vol. 256, (2009) 1514.

[7] Jaeggi, B., Neuenschwander, B., Schmid, M., Muralt, M., Zuercher, J. and Hunziker, U.: Physics Procedia, Vol. 12, (2011) 164

[8] Oztoprak, G., Akman, E., Hanon, M.M., Gnes, M., Gümüs, S., Kacar, E. and Gundogdu, O.: Optics \& Laser Technology, Vol. 45, (2012) 748.

[9] Jandeleit, J., Urbasch G., Hoffmann, H.D., Treusch, H.G. and Kreutz, E.W.: Applied Physics A: Materials Science \& Processing, Vol. 63, (1996) 117.

[10] Raciukaitis, G., Brikas, M., Gecys, P., Gedvilas, M.: Proc. SPIE. Vol. 7005, (2008) 70052L.

[11] Hashida, M., Semerok, A.F., Gobert, O., Petite, G., Iazwa, Y. and Wagner, J.F.: Applied Surface Science, Vol. 197-198, (2002) 862.

[12] Preuss, S., Demchuk, A. and Stuke, M.: Applied Physics A, Vol. 61, (1995) 33.

[13] Chichkov, B.N., Momma, C., Nolte, S., von Alvensleben, F. and Tünnermann, A.: Applied Physics A, Vol. 63, (1996) 109.

(Received: June 30, 2014, Accepted: May 7, 2015) 